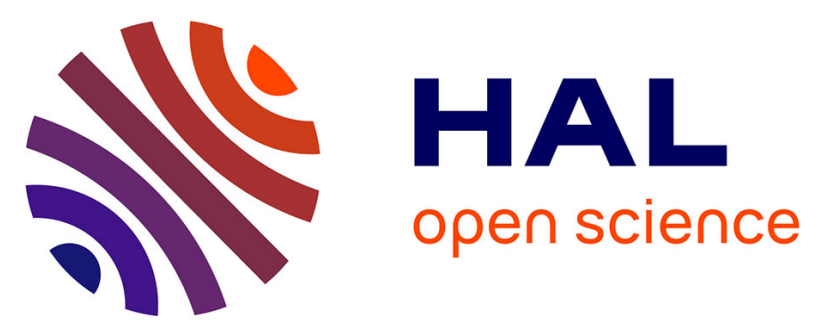

\title{
A Stochastic Theoretical Game Approach for Resource Allocation in Vehicular Fog Computing
}

Habtamu Mohammed Birhanie, Sidi-Mohammed Senouc, Mohammed Ayoub Messous, Amel Arfaoui, Ali Kies

\section{- To cite this version:}

Habtamu Mohammed Birhanie, Sidi-Mohammed Senouc, Mohammed Ayoub Messous, Amel Arfaoui, Ali Kies. A Stochastic Theoretical Game Approach for Resource Allocation in Vehicular Fog Computing. 2020 IEEE 17th Annual Consumer Communications \& Networking Conference (CCNC), Jan 2020, Las Vegas, United States. pp.1-2, 10.1109/CCNC46108.2020.9045224 . hal-03013938

\section{HAL Id: hal-03013938 \\ https://hal.science/hal-03013938}

Submitted on 14 Feb 2022

HAL is a multi-disciplinary open access archive for the deposit and dissemination of scientific research documents, whether they are published or not. The documents may come from teaching and research institutions in France or abroad, or from public or private research centers.
L'archive ouverte pluridisciplinaire HAL, est destinée au dépôt et à la diffusion de documents scientifiques de niveau recherche, publiés ou non, émanant des établissements d'enseignement et de recherche français ou étrangers, des laboratoires publics ou privés. 


\title{
A Stochastic Theoretical Game Approach for Resource Allocation in Vehicular Fog Computing
}

\author{
Habtamu Mohammed Birhanie ${ }^{1,3}$, Sidi-Mohammed Senouci ${ }^{1}$, Mohammed Ayoub Messous ${ }^{1}$, \\ Amel Arfaoui ${ }^{1}$, Ali Kies ${ }^{2}$ \\ ${ }^{1}$ DRIVE EA 1859, Univ. Bourgogne Franche Comté, F58000, Nevers, France. \\ ${ }^{2}$ Unisversity of Science and Technology of Oran, MB, Algeria \\ ${ }^{3}$ Kombolcha Institute of Technology, Wollo University, Kombolcha, Ethiopia \\ habtamu_birhanie@etu.u-bourgogne.fr ; \{Sidi-Mohammed.Senouci, ayoub.messous,amel.arfaoui\}@u-bourgogne.fr, \\ ali.kies@gmail.com
}

Keywords - Vehicular Fog Computing, Resource Allocation, Stochastic Game, Electric Vehicles.

Abstract

\begin{abstract}
Mobile devices have usually limited capabilities in terms of computation power, battery lifetime, storage size and available bandwidth. Thus, to address these limitations and to continue supporting the ever-increasing application requirements, service providers use powerful servers in order to offer services through the cloud. However, due to latency and QoS limitations, cloud computing still does not solve all the problems of newly emerging mobile applications demands. Thus, a more recent development is to push the storage and processing capabilities to the edge of access network closer to end users, which introduce the new concept of fog computing. Fog computing is a decentralized computation framework which essentially extends cloud computing resources and services to the edge of access network [2].

In another context, the global number of EVs has reached above 5 millions on 2018, an increment by 2 millions from 2017 , and this number is predicted to be above 130 millions in 2030 [3]. Moreover, with the advancement of information communication technology in the automotive industries, these EVs are becoming resourceful with more powerful onboard computing, storage, communication capabilities, and with higher battery storage capacity. However, according to [4], more than $95 \%$ of the time these vehicles spent their time in a parking. Hence, we came up with the idea of putting to use abundant and idle resource available within parked EVs for the execution of resource hungry task of nearby mobile user's applications demands.

Some existing works have studied the use of unused resources of parked vehicles in order to provide communication, computation, and to serve end users. In [5], a vehicular cloud computing (VCC) system that coordinates vehicular resources and remote cloud is proposed. However, the recent development is to integrate vehicles' resources with the available resources in nearby which creates a new framework called vehicular fog computing (VFC). For instance, [6] studies the potential gains of using VFC as an infrastructure for communication, computation, and storage. In [7], the authors propose to use VFC as a relaying layer for offloading highly intensive computation tasks from mobile devices.
\end{abstract}

In this paper, we propose to use parked EVs resources for serving local mobile application demands. Hence, unused resources of parked EVs are aggregated in order to provide intime service to mobile devices with constrained energy, limited storage, and processing capabilities. Moreover, the number of mobile users and amount of vehicular fog nodes resources is dynamic over time. For such types of situations, the long-term system performance is more relevant. Therefore, we adopt a game theory approach for resource allocation in VFC. Game theory is a powerful tool to design distributed mechanisms, so that the users of mobile devices can locally make decisions based on strategic interactions and achieve a mutually satisfactory computation offloading solution.

As illustrated in Figure 1, vehicular fog computing architecture is proposed. In the proposed architecture, vehicular fog nodes augment the capabilities of resource constrained mobile devices, by offloading the computation demand via fog controller. Hence, we consider a set of mobile users which execute computationally intensive tasks and delay sensitive tasks while trying to preserve their energy and delay. These mobile users are defined as the players in our game with two possible states (active/inactive) and compete for time varying available resources within parked VFC. In addition, we use

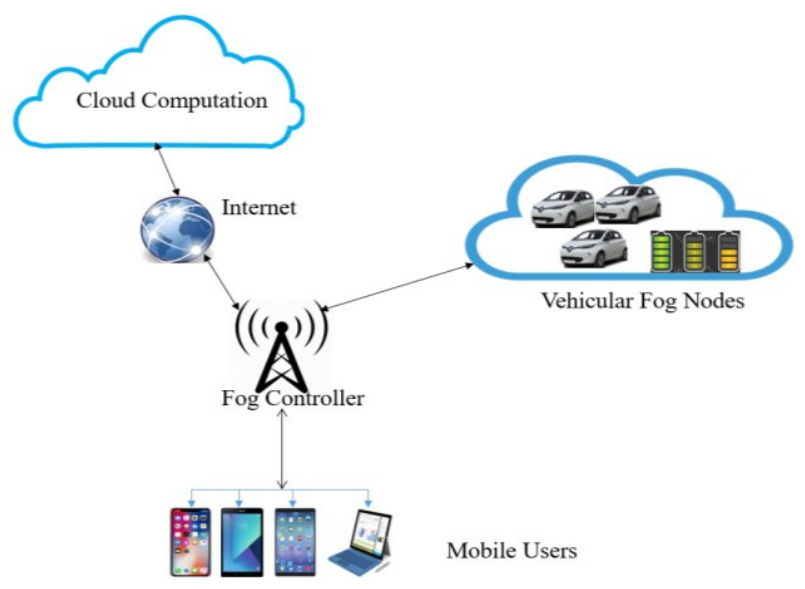

Fig. 1 - Parked Vehicular Fog Computing Architecture 
aggregated resources of parked EVs resources as a vehicular fog node. We also introduced a fog controller through which users can offload their computation tasks to neighboring vehicular fog nodes or towards distant cloud servers. Thus, three strategies are identified for performing the computation, namely computing locally, offloading to the vehicular fog nodes or offloading to the cloud. Therefore, we formulate a new resource allocation problem in a multiuser context based in a computation-offloading scheme. Furthermore, we implement a new compatible algorithm to resolve the problem and reach an equilibrium. Besides, a comprehensive simulation work is achieved in order to prove the effectiveness of the proposed solution.

In the following, we present the performance of resource allocation in parked VFC through simulation using Matlab. In order to conduct the simulations, we setup a scenario where a group of mobile users scattered in the coverage of the fog controller network. Each mobile user may randomly be active or inactive according to a uniform distribution probability within $[0,1]$. Different learning step-sizes for the algorithm are considered a value of 0.1 and 0.5 .

For evaluating the performances of the proposed solution, different performance metrics have been considered:

- The convergence of the proposed algorithm has been evaluated. Thus, we plot the strategy selection probability for different step sizes is evaluated.

- The overall system wide cost, which represents the total computation overhead of the system, is evaluated.

First, to evaluate the convergence of the proposed solution, we plot the average strategy selection probabilities for 25 selected users with different learning step-sizes. Step-size influences the convergence time of the algorithm and has greater impact on the oscillation behavior of the graphs. As shown in Figure 2, with a smaller step-size $(b=0.1)$, the users select their strategy from uniform probability distribution. However, when it continues to use the proposed algorithm, they keep updating their strategy selection probability and did not converge for our simulation time.

However, in Figure 3, we use a step-size $b=0.5$. Initially, the user's mobile selects their offloading strategy according to the probability distribution then the user's strategy selection probability is keep updating. Finally, the probability to choose offloading strategy to VFC converges to 1 . This result shows that, after convergence mobile users choose VFC to perform their computation tasks.

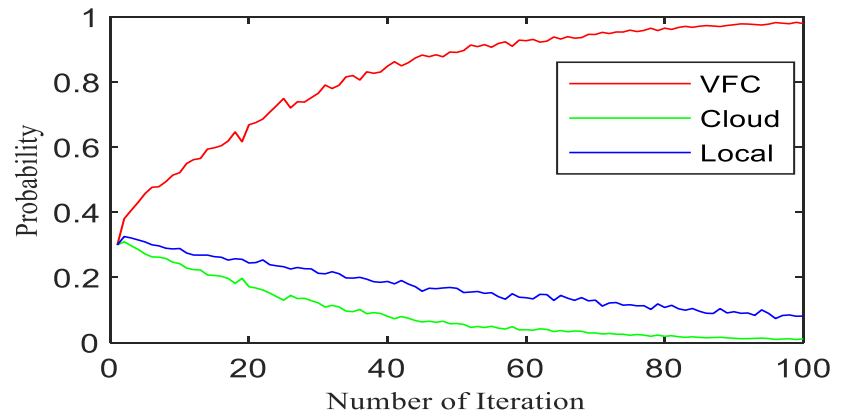

Fig. 2 - Strategy selection probability when $b=0.1$

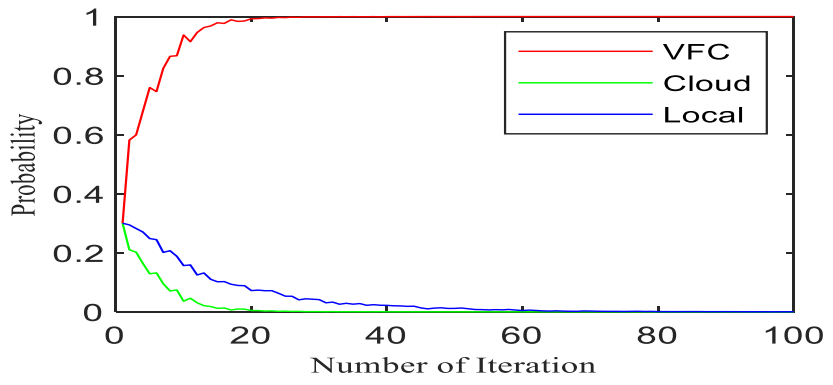

Fig. 3 - Strategy selection probability when $b=0.5$

Figure 4 shows the system wide cost for the two step-sizes. As it is shown, the cost of offloading tasks at $b=0.1$ is lower than $b=0.5$. This is because the convergence speed is slow when the value of $b$ decreases and takes more time to converge.

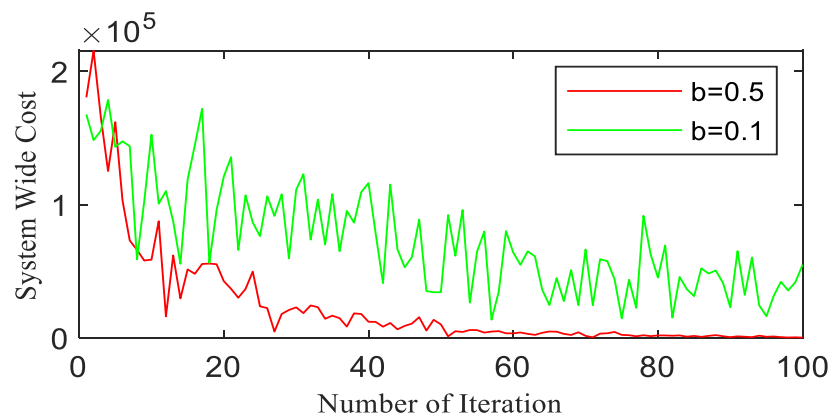

Fig. 4 - System Wide Cost for $\mathrm{N}=10$ mobiles users use case

In this paper, we propose to use resources of parked EVs as fog nodes, in order to extend the computation capabilities of constrained neighboring mobile devices. The amount of available resources in vehicular fog nodes and the number of active computation demands are highly dynamic. Therefore, a stochastic theoretical game has been formulated, with the objective to minimize energy and delay overhead. Finally, the obtained simulation results show the effectiveness of the proposed solution compared to classical models.

\section{REFERENCES}

[1] H. M. Birhanie, M. A. Messous, S. Senouci, E. Aglzim, and A. M. Ahmed, "MDP-based Resource Allocation Scheme towards a Vehicular Fog Computing with Energy Constraints," 2018 IEEE Glob. Commun. Conf., pp. 1-6, 2018.

[2] S. Yi, Z. Hao, Z. Qin, and Q. Li, "Fog computing: Platform and applications," Proc. - 3rd Work. Hot Top. Web Syst. Technol. HotWeb 2015, pp. 73-78, 2016.

[3] Till Bunsen et al., "Global EV Outlook 2019 to electric mobility," OECD iea.org, p. 232, 2019.

[4] D. T. Hoang, P. Wang, D. Niyato, and E. Hossain, "Charging and discharging of plug-in electric vehicles (PEVs) in vehicle-to-grid (V2G) systems: A cyber insurance-based model," IEEE Access, vol. 5, pp. 732754, 2017.

[5] C. C. Lin, D. J. Deng, and C. C. Yao, "Resource Allocation in Vehicular Cloud Computing Systems with Heterogeneous Vehicles and Roadside Units," IEEE Internet Things J., vol. 5, no. 5, pp. 3692-3700, 2018.

[6] S. Raza, S. Wang, M. Ahmed, and M. R. Anwar, "A Survey on Vehicular Edge Computing: Architecture, Applications, Technical Issues, and Future Directions," vol. 2019. pp. 1-19, 2019.

[7] Z. Wang, Z. Zhong, S. Member, and D. Zhao, "Vehicle-Based Cloudlet Relaying for Mobile Computation Offloading," IEEE Trans. Veh. Technol., vol. PP, no. c, p. 1, 2018. 